\title{
Estimación de la edad cronológica en función de la mineralización del tercer molar inferior en población andina
}

Estimation of the chronological age based on the mineralization of the third lower molar in the andean population

Andrea Rodríguez ${ }^{1}$, Verónica Verdugo ${ }^{2, a}$, Guisela Loarte ${ }^{1}$, Ebingen Villavicencio ${ }^{2, a}$, Esteban Torracchi ${ }^{2, a}$.

\section{RESUMEN}

Objetivo: Determinar el nivel de mineralización del tercer molar inferior de acuerdo a la edad cronológica mediante el método Demirjian en radiografías panorámicas digitales de pacientes andinos. Material y métodos: Se realizó un estudio correlacional analizando 706 radiografías panorámicas digitales en la Unidad Académica de Salud y Bienestar de la Universidad Católica de Cuenca Ecuador, en el período 2015-2016. Los pacientes tuvieron entre 6 y 22 años de edad. Se Evaluó un total de 1412 terceros molares (4.8 y 3.8). Resultados: Un primer análisis descriptivo mostró una correlación fuerte $(\mathrm{Rho}=0,99)$ entre la edad cronológica y el nivel o grado de mineralización del tercer molar inferior. En ambas piezas estudiadas el inicio de la formación del tercer molar se dio en promedio a los 8,13 años y finalizaba a los 20,41 años. Los estadios que mostraron mayor coincidencia en ambos sexos y en ambas piezas fueron B, C, F, G y $\mathrm{H}$ y los estadios con menor coincidencia fueron los estadios A, D, E. Conclusiones: De acuerdo a los resultados podemos sugerir que el método Demirjian, es una herramienta útil para determinar la edad cronológica en base a la mineralización de los terceros molares, con un error de $+/-1.2$ meses por lo que es posible ser usado para la estimación de la edad en evaluaciones forenses.

PALABRAS CLAVE: Calcificación, tercer molar, radiografía, cronología. 


\section{SUMMARY}

Objective: To determine the level of mineralization of the third lower molar according to chronological age using the Demirjian method in panoramic digital radiographies of Andean patients. Material and methods: A correlational study was carried out analyzing 706 digital panoramic radiographies in the Unidad Académica de Salud y Bienestar de la Universidad Católica de Cuenca Ecuador, in the period 2015-2016. The patients were between 6 and 22 years of age. A total of 1412 third molars (4.8 and 3.8) were evaluated. Results: A first descriptive analysis showed a strong correlation $(\mathrm{Rho}=0.99)$ between chronological age and the level or degree of mineralization of the lower third molar. In both studied pieces the beginning of the formation of the third molar was given in average at 8,13 years and finished at 20,41 years. The stages that showed greater coincidence in both sexes and in both pieces were B, C, F, G and $\mathrm{H}$ and the stages with less coincidence were the stages A, D, E. Conclusions: According to the results we can suggest that the Demirjian method is a useful tool to determine the chronological age based on the mineralization of the third molars, with an error of $+/-1.2$ months so it is possible to be used for the estimation of the age in forensic evaluations.

KEYWORDS: calcification, third molar, radiography, chronology.

\section{INTRODUCCIÓN}

La edad cronológica de un individuo se define como el tiempo transcurrido desde su nacimiento, hasta el momento que se requiera su estimación o hasta su muerte (1). En circunstancias en que así se requiera (crímenes, masacres, migraciones masivas, etc.), esta edad se puede estimar a través de parámetros morfológicos, anatómicos o fisiológicos como el sexo, o la maduración ósea como resultado del proceso de crecimiento y desarrollo de los huesos (2). En el caso de los dientes, según Cadenas y cols. la maduración dental se correlaciona con los estadios de mineralización, de forma tal que se puede estimar de manera indirecta, y mediante análisis radiográfico, cuál es la edad aproximada de una persona de acuerdo con el nivel de mineralización de sus dientes (3). Se trata de una estimación fiable y conveniente, pues las estructuras dentales mineralizadas forman parte del componente indestructible del cuerpo humano, siendo objeto de muy pocos cambios de su estructura original y conservándose sin modificaciones por muchos años $(4,5)$.

En efecto, la mineralización de los dientes evoluciona con una secuencia invariable que va desde las puntas de las cúspides, luego procede con la formación de la corona, posteriormente con el desarrollo de la raíz y finalmente termina con el cierre apical. Esta maduración culmina durante la adolescencia tardía; sin embargo, el tercer molar es la única pieza dentaria que continua con su formación después de la adolescencia $(2,6)$. Dada la ubicación anatómica de los terceros molares, es posible pensar que son las piezas dentales más adecuadas para realizar este tipo de estudios; por otra parte, su tardía aparición en la boca permite tener presente a esta pieza por mucho más tiempo que las otras molares.

Hoy en día, la mayoría de los métodos empleados para estimar la edad de las personas, consumen mucho tiempo, son costosos y requieren de personal calificado. Los exámenes radiográficos complementarios son un recurso confiable que pueden ser empleados para observar la estructura dental, proporcionan datos que permiten determinar la edad aproximada de niños y adultos. Estas radiografías también permiten estimar el grado de maduración de los dientes y su estadio de mineralización. Uno de los métodos más utilizados es el propuesto por Demirjian (7), que clasifica la mineralización de las piezas dentarias en 8 estadios codificados desde la A hasta la $\mathrm{H}(8,9,10)$ (figura $1)$.

La mayoría de los estudios realizados en Iberoamérica publicados hasta la fecha se han llevado a cabo en países como España (1), Brasil (11) y Perú (12). En el caso de Ecuador, son muy escasos los estudios en este sentido. Por tal razón el objetivo de la presente investigación fue establecer los valores promedio de la edad cronológica en cada estadio de maduración del tercer molar inferior de individuos de la Sierra Ecuatoriana, a partir de las radiografías panorámicas digitales analizadas mediante el método Demirjian. 


\section{MATERIAL Y MÉTODOS}

En el presente estudio correlacional, se emplearon 706 radiografías panorámicas disponibles en el archivo digital del área de rayos X de la Universidad Católica de Cuenca, que proporcionaron 1412 terceros molares para ser estudiados. Se incluyeron radiografías pertenecientes a pacientes, cuyas edades variaban entre los 6 y 22 años, y la presencia de los terceros molares inferiores orientados en cualquier dirección. Para cada paciente, se registró el estadio de los dos terceros molares inferiores (piezas 4.8 y 3.8 ) (7).

Los estadios según la clasificación de Demirjian son los siguientes: "A (calcificación inicial de la corona); $\boldsymbol{B}$ (superficie oclusal fusionada); $\boldsymbol{C}$ (superficie oclusal formada); $\boldsymbol{D}$ (formación de la corona completa hasta la unión esmalte cemento); $\boldsymbol{E}$ (bifurcación radicular visible); $\boldsymbol{F}$ (longitud radicular igual al largo de la corona); $\boldsymbol{G}$ (ápice de los canales radiculares parcialmente abierto) y $\boldsymbol{H}$ (ápice de los canales radiculares cerrados)" (7).

Los evaluadores que participaron en el estudio fueron calibrados por una experta, obteniendo un valor de concordancia Kappa $>0,8$. Los datos fueron analizados mediante estadística descriptiva (media, desviación estándar, valor mínimo, valor máximo) a continuación se evaluó la correlación entre la edad cronológica y los estadios de maduración utilizando la prueba Rho de Spearman, también se realizó la correlación entre los estadios de ambas piezas y finalmente se calculó el error estándar para el 95\% de confiabilidad en cada uno de los estadios y para el total de los datos.

Tabla 1. Correlación de la edad cronológica de los terceros molares inferiores, de acuerdo los estadios Demirjian según el sexo.

\begin{tabular}{|c|c|c|c|c|c|c|c|c|c|c|c|c|c|}
\hline & \multicolumn{8}{|c|}{ SEXO } & \multirow{2}{*}{\multicolumn{3}{|c|}{ TOTAL }} & \multirow{2}{*}{\multicolumn{2}{|c|}{ Rho }} \\
\hline & \multicolumn{4}{|c|}{ FEMENINO } & \multicolumn{4}{|c|}{ MASCULINO } & & & & & \\
\hline & Media & D.E. & Mínimo & Máximo & Media & D.E. & Mínimo & Máximo & Media & D.E. & Mínimo & Máximo & Spearman \\
\hline \multicolumn{14}{|c|}{$\begin{array}{c}\text { ESTADIOS } \\
4.8\end{array}$} \\
\hline & & & & & & & & & & & & & $0,801 *$ \\
\hline A & 7,95 & 2,02 & 6 & 15 & 8,13 & 1,62 & 6 & 14 & 8,03 & 1,85 & 6 & 15 & $\begin{array}{c}0,746 \\
\text { entre sexos }\end{array}$ \\
\hline B & 8,45 & 1,63 & 6 & 11 & 8,25 & 1,37 & 6 & 11 & 8,37 & 1,52 & 6 & 11 & \\
\hline $\mathrm{C}$ & 12,87 & 2,26 & 10 & 18 & 12,12 & 2,15 & 10 & 18 & 12,47 & 2,2 & 10 & 18 & \\
\hline $\mathrm{D}$ & 16,4 & 2,81 & 10 & 22 & 15,86 & 2,52 & 10 & 21 & 16,17 & 2,69 & 10 & 22 & \\
\hline $\mathrm{E}$ & 18,13 & 2,62 & 13 & 22 & 17,61 & 2,04 & 15 & 21 & 17,96 & 2,43 & 13 & 22 & \\
\hline $\mathrm{F}$ & 18,19 & 2,12 & 10 & 21 & 18 & 1,63 & 15 & 20 & 18,14 & 2 & 10 & 21 & \\
\hline G & 19,71 & 1,37 & 16 & 22 & 19,8 & 1,49 & 15 & 22 & 19,75 & 1,43 & 15 & 22 & \\
\hline $\mathrm{H}$ & 20,15 & 1,16 & 17 & 22 & 20,41 & 1,27 & 17 & 22 & 20,3 & 1,23 & 17 & 22 & \\
\hline \multicolumn{14}{|c|}{$\begin{array}{c}\text { ESTADIOS } \\
3.8\end{array}$} \\
\hline & & & & & & & & & & & & & $0,801^{*}$ \\
\hline A & 7,95 & 2,02 & 6 & 15 & 8,1 & 1,64 & 6 & 14 & 8,01 & 1,86 & 6 & 15 & $\begin{array}{c}0,750 \\
\text { entre sexos }\end{array}$ \\
\hline B & 8,45 & 1,63 & 6 & 11 & 8,29 & 1,35 & 6 & 11 & 8,38 & 1,51 & 6 & 11 & \\
\hline $\mathrm{C}$ & 12,87 & 2,26 & 10 & 18 & 12,28 & 2,19 & 10 & 18 & 12,55 & 2,21 & 10 & 18 & \\
\hline $\mathrm{D}$ & 16,4 & 2,81 & 10 & 22 & 15,98 & 2,59 & 10 & 21 & 16,22 & 2,72 & 10 & 22 & \\
\hline $\mathrm{E}$ & 18,09 & 2,63 & 13 & 22 & 17,5 & 2,02 & 15 & 21 & 17,89 & 2,44 & 13 & 22 & \\
\hline $\mathrm{F}$ & 18,23 & 2,12 & 10 & 21 & 18 & 1,63 & 15 & 20 & 18,17 & 2 & 10 & 21 & \\
\hline G & 19,71 & 1,37 & 16 & 22 & 19,81 & 1,48 & 15 & 22 & 19,76 & 1,42 & 15 & 22 & \\
\hline $\mathrm{H}$ & 20,15 & 1,16 & 17 & 22 & 20,41 & 1,28 & 17 & 22 & 20,29 & 1,23 & 17 & 22 & \\
\hline
\end{tabular}




\section{RESULTADOS}

La muestra obtenida fue de 1412 piezas que provenían de 706 radiografías panorámicas de pacientes entre 6 y 22 años de edad, 391 mujeres y 315 varones. Los resultados de este estudio indican que el inicio de la mineralización de la pieza 4.8 se da a la edad promedio de 8.03 años (calificación inicial de las coronas); luego continúa con la mineralización de las superficies oclusales a los 8.37 años ; a los 12,47 años se da la formación completa de la superficie oclusal; a los 16.17 años la corona está casi completa, a los 17,96 años la superficie radicular es visible; a los 18,14 años la longitud radicular es igual al largo de la corona; a los 19,75 años se encontraba el ápice radicular parcialmente abierto; y por último a los 20,3 se encontraba el ápice de los canales radiculares cerrado (tabla 1).

En la pieza 3.8 la edad promedio del inicio de la mineralización es de 8,01 años estadio A; a los 8.38 años se da el estadio B; a los 12,55 años estadio C; a los 16.22 estadio D, a los 17,89 años estadio E; a los 18,17 estadio F; a los 19.76 años estadio G; y por último a los 20,29 años se encontraba el estadio $H$ (tabla 1).
Al correlacionar la edad cronológica con la mineralización del tercer molar inferior destaca la correlación fuerte que existe entre estas dos variables; correlación total entre estadios (Rho Spearman $=0,801$ en mujeres y varones) y correlación total entre piezas (Rho Spearman= 0,999). Por otro lado, podemos destacar que en los resultados obtenidos se encuentra exactamente el mismo valor de correlación (entre la edad y los estadios) tanto en la pieza 3.8 como en la 4.8; de igual forma se encontró también datos muy similares en la correlación de valores de estadios entre varones y mujeres. La mayor coincidencia de las edades de una y otra pieza se dio en los estadios B, C, F, G, H y los estadios con menor coincidencia son A, D y E (tabla 1).

\section{DISCUSIÓN}

En el presente estudio el objetivo principal fue determinar la edad promedio en cada uno de los estadios de mineralización de los terceros molares inferiores y la edad cronológica de habitantes de la Sierra Ecuatoriana, utilizando para tal fin radiografías panorámicas digitales. Los resultados de nuestro estudio indican que existe
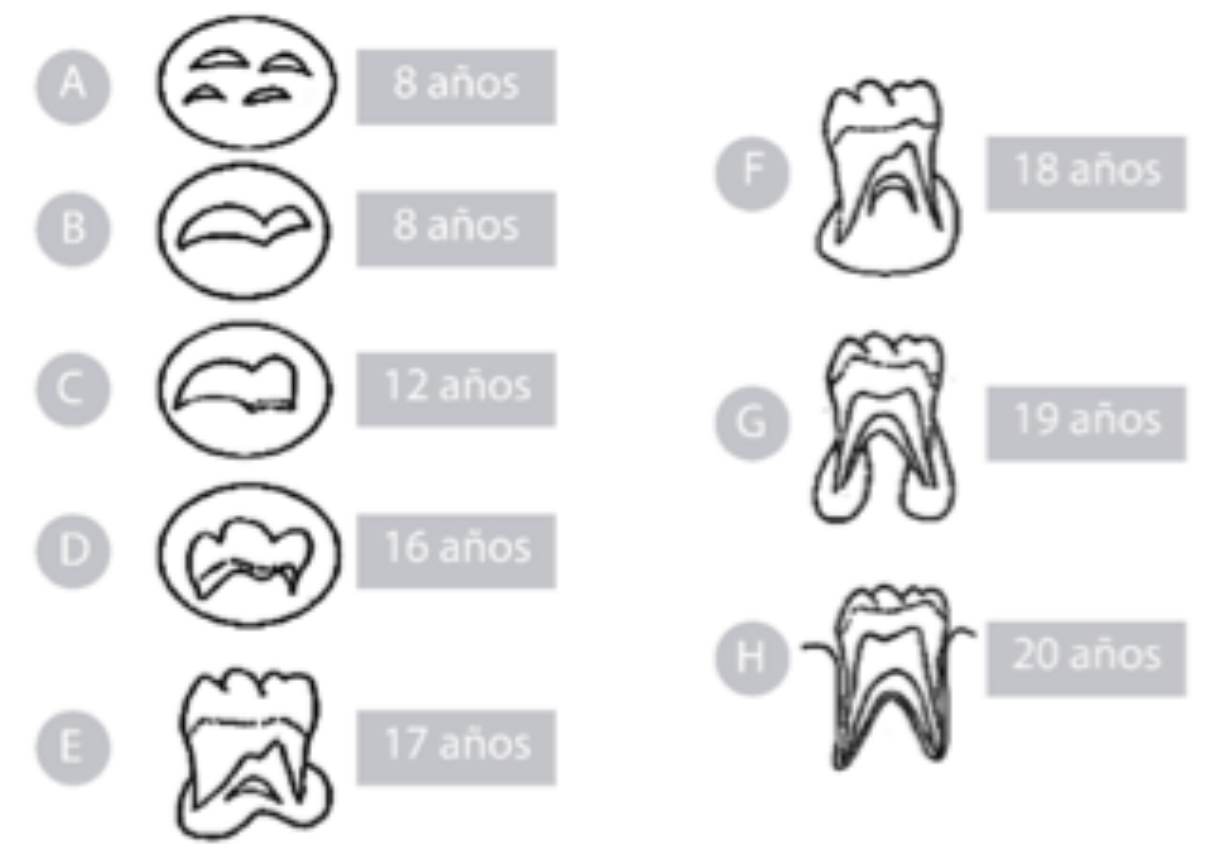

Fuente: Elaborado por autores.

Figura 1. Valores totales de estadios Demirjian en las piezas 3.8; 4.8 
una correlación muy alta entre ambas variables y, por lo tanto, confirman la utilidad de este método para distintos fines, desde medicina forense hasta estudios antropológicos.

El método Demirjian fue inicialmente propuesto por su autor como un examen objetivo y de fácil aplicación $(13,14)$, desde entonces, diversos autores han confirmado la utilidad de este método para estimar la edad cronológica de los pacientes a partir de la determinación del estadio de mineralización en el que se encuentran los primeros y segundos molares. Sin embargo, se ha demostrado que existen variaciones que dependen de las características genéticas (etnia), nutricionales, ambientales, por lo cual se ha insistido en la necesidad de adaptar estos valores a las características particulares de distintos grupos humanos (6). Este es el caso que se presenta para comunidades de la región andina Suramericana, donde hay muy pocos reportes de estudios sobre la aplicación del Método Demirjian en terceros molares inferiores para estimar la edad cronológica (12). Los resultados obtenidos en nuestro estudio dan a conocer la relación que existe entre los estadios Demirjian y el grado de mineralización dental, donde se puede observar que la mineralización de la pieza 4.8 inicia a los 8,03 años y finaliza a los 20,3 donde los ápices de los canales radiculares están cerrados. Estos resultados son significativamente similares en la pieza 3.8.

Domínguez realizó un estudio en pacientes de 7 a 23 años de edad que acudieron al centro radiológico privado de Azogues Cuenca-Ecuador, evaluaron 596 radiografías panorámicas y concluyeron que los terceros molares se pueden considerar como estimadores de la edad (15), sus resultados difieren con el presente estudio; en la pieza 3.8; dado que ellos presentan que en el sexo femenino inicia la mineralización a los 9,15 años y finaliza a los 21,19 años; en el sexo masculino inicia a los 10,00 años y finaliza a los 21,02 años; mientras que en el presente estudio las edades de inicio son más tempranas y las edades de finalización también son menores. En el estudio de Domínguez la pieza 4.8 en el sexo femenino inicia a los 9,50 años y finaliza a los 21,01 años por tal razón no encontraron diferencias estadísticamente significativas en los estadios de Demirjian entre hombres y mujeres (15). Esto puede deberse al tipo de alimentación que presenten los individuos de esta población perteneciente al Cantón Azogues que involucran diferentes factores socioeconómicos.

Quezada y col. evaluaron 1176 radiografías panorámicas con presencia de terceros molares inferiores en Perú en pacientes de la Universidad Peruana Cayetano Heredia entre 7 y 23 años de edad, en su estudio concluyeron que se presentó una maduración mayor en el tercer molar inferior en el sexo masculino que en el femenino (12), dato que difiere con los hallazgos del presente estudio; también nos revelan que en el estadio D la edad promedio es de 14,05 en mujeres mientras que en este mismo estadio el promedio para varones es de 13,42 años. También reportan que se encontró una diferencia significativa en los estadios C, D, E, F, G, H y con menor coincidencia en los estadios A y $\mathrm{B}$, datos que difieren con nuestro estudio en cuanto a la concordancia entre estadios (12). Como una posible explicación puede deberse a los factores geográficos y socioeconómicos entre estas dos poblaciones.

Prieto y col., realizaron un estudio para calcular la estimación de la edad cronológica determinada de acuerdo a las etapas de desarrollo del tercer molar inferior. La muestra fue de 1054 radiografías panorámicas de pacientes que tenían entre $14 \mathrm{y}$ 21 años; 592 del sexo femenino y 462 del sexo masculino, en una población española (1). Sus resultados determinan que existe mayor correlación en los hombres ya que la formación de la corona y raíz finalizó antes que en las mujeres. La diferencia promedio entre la edad cronológica y la edad estimada fue de - 0,10 años para la pieza 3.8 y - 0,07 años para la pieza 4.8; con cierta variación en lo que respecta al sexo (1), dato que no coincide con los resultados del presente estudio podría deberse, a factores étnicos y factores geográficos entre estas dos poblaciones.

Toledo evaluó a 407 radiografías panorámicas en un estado de Brasil en pacientes de ambos sexos, con la finalidad de evaluar la cronología de la mineralización del tercer molar y si esta podría ser un indicador preciso para medir la edad, concluyó que los hombres tenían etapas de mineralización más progresiva que las mujeres (11); sin embargo, no había una fuerte correlación entre la edad y la etapa de mineralización entre en el sexo masculino y femenino, encontró que 
el 96,8-98,6\% de los pacientes se encontraban entre los 18 años o más en el estadio $\mathrm{H}$, estos resultados difieren de lo presentado, posiblemente por el período de elaboración entre los estudios sobre el desarrollo dental y por factores étnicos.

\section{CONCLUSIONES}

De acuerdo a los resultados podemos sugerir que el método Demirjian, es una herramienta útil para determinar la edad cronológica en función a la mineralización de los terceros molares. Los resultados de esta investigación mostraron un margen de error de $+/-1.2$ meses. Finalmente es posible que estas piezas dentarias sean de gran importancia ya que son los últimos dientes en erupcionar después de la adolescencia y tienen alta probabilidad de mantenerse en boca en edades avanzadas. Teniendo en cuenta que en la población ecuatoriana la mineralización completa del tercer molar se observa a partir de los 20 años en ambos sexos.

Agradecimientos: Los autores agradecen al Dr. Luis Andrés Yarzábal (Departamento de Investigación, Carrera de Odontología Universidad Católica de Cuenca) por su apoyo en la edición y revisión crítica de este manuscrito.

\section{Correspondencia:}

Guisela Loarte

Correo electrónico:guise_9218@hotmail.com

\section{REFERENCIAS BIBLIOGRAFICAS}

1. Prieto J, Barberia E, Ortega R. Evaluation of chronological age based on third molar development in the Spanish population. Int J Legal Med 119(6):349-54.

2. Cardenas I, Celis C, Hidalgo A, Schiling A. Estimación de Edad Dentaria Utilizando el Método de Demirjian en Niños de 5 a 15 Años de Curicó, Chile. Int. J. Odontostomat. 2014; 8(3):453-459.

3. Cadenas I, Celis C, Hidalgo A. Método de Demirjian para estimación de edad dentaria en base a estadios de mineralización. Anu Soc Radiol Oral Máxilo Fac Chil. 2010; 13:17-23.

4. Espinoza A. Relación entre la edad dental utilizando el método de demirjian y la edad cronológica en una población de 4 a 16 años. Revista Cientifica Salud y vida Sipanese. 2016; 3(2):21-28.

5. Hernández Z, Acosta M. Comparación de Edad Cronológica y Dental según Índices de Nolla y Dermijian en pacientes con acidosis tubular renal. Pesquisa Brasileira em Odontopediatria e Clínica Integrada. 2010; 10(3): 423-431.

6. Ortega A, Martínez V. Estimación de la edad dental por el método de Demirjian y sus modificaciones en un grupo de Venezolanos. ODOUS CIENTIFICA. 2015; 16(1): 9-17.

7. Demirjian A, Goltein H, Tanner KM. A New Sistem of dental age assessment. Hum Biology. 1973: 45(2):211-227.

8. Perdomo M. Estimación de la edad cronológica a partir del desarrollo dentario. Tesis Doctoral. Valencia: Facultad de Medicina y Odontología; 2014.

9. Pizarro M, Quezada M, Del Castillo C, Orejuela F. Estimación de la edad de acuerdo al método de Demirjian en niños de 4 a 16 años de la Ciudad de Puebla. Revista Estomatológica Herediana. 2016; 26(3): 139-46.

10. Prieto J. La maduración del tercer molar y el diagnóstico de la edad. Evolución y estado actual de la cuestión. Cuad Med Forense. 2008;14(51): 11-24.

11. Toledo F. Mineralization of mandibular third molars can estimate chronological age-Brazilian indices. Forensic science international. 2012; 219(1-3): 147150.

12. Quezada M, Beltrán J, Bernal J, Alva E, Castillo C. Relación entre la edad cronológica y la mineralización del tercer molar inferior segun método Demirjian. Rev Estomatol Herediana. 2014: 24(2):63-72. DOI: https:// doi.org/10.20453/reh.v24i2.2126

13. Marañón G, Gonzales H. Edad dental según los métodos Demirjian y Nolla en niños peruanos de 4 a 15 años. Kiru. 2012: 9(1): 1-9.

14. Ramirez J, Garza A, Moreno E, Verdugo M, Lopez J, Garnica J. Concordancia entre la Edad Cronológica y Edad Dental Según el Método de Demirjian en Pacientes Mexicanos. Int J Odontostomat. 2018; 12(4): 412-415.

15. Domínguez D. Relación entre la edad cronológica y estadios de mineralización del tercer molar inferior en radiografías panorámicas digitales de pacientes entre 7 y 23 años que acudieron a centro radiológico privado. Tesis. Lima, Perú: Universidad Peruana Cayetano Heredia; 2017.

Recibido : 18-08-2020

Aceptado : 17-12-2020 